\title{
Quasielastic neutron scattering study of POSS ligand dynamics
}

\author{
Niina Jalarvo ${ }^{1,2, a}$, Madhusudan Tyagi ${ }^{3,4}$ and Michael K. Crawford ${ }^{5}$ \\ ${ }^{1}$ Jülich Centre for Neutron Science, Forschungszentrum Jülich GmbH, 52428 Jülich, Germany \\ ${ }^{2}$ Chemical and Engineering Materials Division, Spallation Neutron Source, Oak Ridge National Laboratory, Oak Ridge, \\ TN 37861-6475, USA \\ ${ }^{3}$ NIST Center for Neutron Research, Gaithersburg, MD 20899-8562, USA \\ ${ }^{4}$ Department of Materials Science, University of Maryland, College Park, MD 20742, USA \\ ${ }^{5}$ DuPont Central Research and Development, Wilmington, DE 19880-0400, USA
}

\begin{abstract}
Polyoligosilsesquioxanes are molecules having cage-like structures composed of silicon and oxygen. These molecules can have a wide variety of functional ligands attached to them. Depending on the nature of the ligand, interesting properties and applications are found. In this work we present results from quasielastic neutron scattering measurements of four different POSS molecules that illustrate the presence of strong coupling between the ligand dynamics and the POSS crystal structures.
\end{abstract}

\section{Introduction}

Polyoligosilsesquioxanes (POSS) are relatively large (1-2 nm diameter) molecules, that are composed of $\mathrm{Si}_{8} \mathrm{O}_{12}$ cages to which a wide variety of possible ligands can be attached (Fig. 1). Depending on the nature of the ligand, interesting properties and application possibilities are found, e.g. selective solubility in solvents, the ability to be dispersed in polymers, the catalytic activity and use as fillers in polymer nanocomposites. Thus, the POSS molecules have attracted much interest recently. Structures of more than 100 POSS molecules have been determined using X-ray diffraction [1], but only a few studies of the dynamical properties of the ligands have been reported $[2,3]$. We have studied the ligand dynamics of several POSS molecules using quasielastic incoherent neutron scattering (QENS) techniques. In this paper we will compare the ligand dynamics of the 4 different POSS molecules listed in Table 1. These POSS molecules were chosen for this study because, except for M-POSS, they each exhibit interesting structural phase transitions, which are, as we will show in this paper, connected to the ligand dynamics. Therefore, the intermolecular interactions depend upon the POSS ligand dynamics. Study of the POSS ligand dynamics using quasielastic neutron scattering is thus expected to provide insight regarding the intermolecular interactions that lead to structural phase transitions in POSS and other crystals.

\section{Experimental}

The samples were obtained from Hybrid Plastics and used without further processing. X-ray powder diffraction and NMR were used to confirm the phase purity. QENS data were collected at the backscattering spectrometer BASIS at the Spallation Neutron Source (SNS) [4] and

\footnotetext{
${ }^{a}$ Corresponding author: $n \cdot$ jalarvo@fz-juelich.de
}

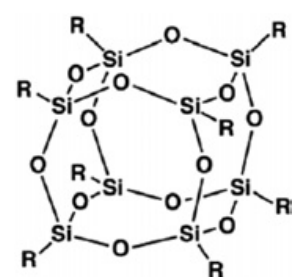

Figure 1. Structure of a POSS molecule. A wide variety of $\mathrm{R}$ groups can be attached to the Si-O framework.

at the high flux backscattering spectrometer (HFBS) at the NIST Center for Neutron Research [5]. The energy resolution at the elastic line (FWHM) was 3.4 and $0.8 \mu \mathrm{eV}$ and the dynamic range \pm 100 and $\pm 17 \mu \mathrm{eV}$ at BASIS and at HFBS, respectively. Cylindrical annular aluminium sample containers were chosen with sample thicknesses giving neutron transmission probability on the order of 90 to $95 \%$. Data sets were collected at temperatures ranging from 4 to $425 \mathrm{~K}$. Low temperature data was used to determine the instrumental resolution function for each sample. In addition data were collected in fixed window mode at HFBS to measure the elastic scattering as a function of temperature.

\section{Results and discussion}

An overview of the microscopic dynamics as a function of temperature can be obtained from the magnitude of the elastic incoherent neutron scattering (EINS). The EINS reflects exclusively hydrogen dynamics, since all of the other elements in the samples have negligible incoherent neutron scattering cross-sections. The EINS as a function of temperature for each sample was obtained using the HFBS spectrometer by switching the Doppler drive off and using the monochromatic beam of $6.27 \AA$ wavelength. An important quantity derivable from the EINS data

This is an Open Access article distributed under the terms of the Creative Commons Attribution License 4.0, which permits unrestricted use, distribution, and reproduction in any medium, provided the original work is properly cited. 
Table 1. The POSS molecules studied in this work, their chemical formulae, the acronyms used and the space groups [1].

\begin{tabular}{|l|l|l|l|}
\hline Acronym & Molecular name & Chemical formula & Crystal Structure \\
\hline M-POSS & Octamethyl-POSS & $\mathrm{Si}_{8} \mathrm{O}_{12}\left(\mathrm{CH}_{3}\right)_{8}$ & rhombohedral R-3 \\
\hline IBU-POSS & Octaisobutyl-POSS & $\mathrm{Si}_{8} \mathrm{O}_{12}\left(\mathrm{C}_{4} \mathrm{H}_{9}\right)_{8}$ & monoclinic/triclinic $\mathrm{T}<330 \mathrm{~K}$, rhombohedral T $>330 \mathrm{~K}$ \\
\hline TMS-POSS & Octatrimethylsiloxy-POSS & $\mathrm{Si}_{8} \mathrm{O}_{12}\left(\mathrm{OSiC}_{3} \mathrm{H}_{9}\right)_{8}$ & Triclinic P-1 $(\mathrm{T}=295 \mathrm{~K})$ \\
\hline DMS-POSS & Octadimethylsilane-POSS & $\mathrm{Si}_{8} \mathrm{O}_{12}\left(\mathrm{OSiHC}_{2} \mathrm{H}_{6}\right)_{8}$ & Rhombohedral R-3 $(\mathrm{T}=200 \mathrm{~K})$ \\
\hline
\end{tabular}
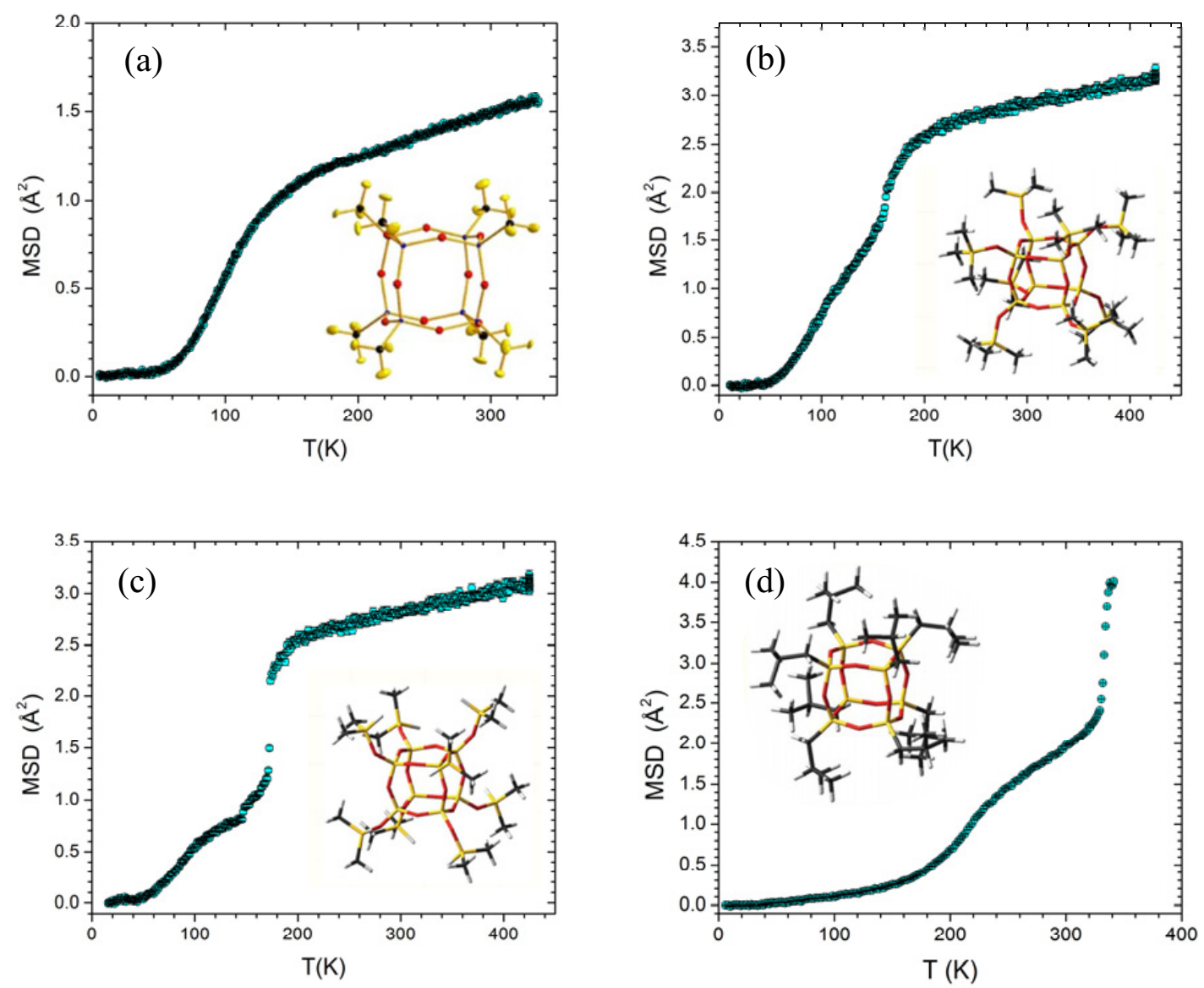

Figure 2. Mean square displacements of (a) M-POSS, (b) TMS-POSS, (c) DMS-POSS and (d) IBU-POSS presented as a function of temperature. Error bars in figures throughout the text represent one standard deviation and if not shown are smaller than the symbols.

is the average mean-square displacement (MSD) of the hydrogen atoms, $<\Delta r^{2}>$, which can be estimated using the Gaussian approximation

$$
I_{e l}(Q, T) / I_{e l}\left(Q, T_{\min }\right)=e^{-Q^{2}\left\langle\Delta r^{2}\right\rangle / 3} .
$$

Equation (1) was fit to the data, and the resulting MSD values for each sample are illustrated in Fig. 2. This approach assumes that all the hydrogen atoms in the material have the same isotropic MSD, which is not exactly the case for the samples of interest here. Therefore, these values only represent the average MSD for all the hydrogen atoms in each sample.

Figure $2 \mathrm{a}$ shows the MSD for M-POSS. At temperatures below $60 \mathrm{~K}$, the MSD follows the expected Debye-Waller type behavior due to harmonic vibrations. Above $60 \mathrm{~K}$ the methyl rotations become observable in the measured time scale, and thus the MSD values start to increase rapidly. We have shown in our previous work
[6] that for M-POSS the methyl groups rotate around local 3 -fold axes, and this is indeed the only motion that is identified in the QENS spectra. However, the other three samples present much more complex dynamical features over the measured temperature range.

Figure $2 b$ illustrates the obtained MSD values for TMS-POSS. The low temperature MSD features are very similar to those of M-POSS up to about $140 \mathrm{~K}$. This indicates that in TMS-POSS the methyl groups rotate first around their local 3-fold axes, but at temperatures above $140 \mathrm{~K}$ different dynamical processes take over. As also observed for M-POSS the rapidly increasing MSD values start to flatten above $140 \mathrm{~K}$, but then there is a second rapid increase of MSD values for TMS-POSS. Near $170 \mathrm{~K}$ a discontinuity in the MSD values is observed, suggesting the presence of a first-order structural phase transition. These large MSDs are beyond the values that can be expected for lone methyl group rotations, and most likely the three methyl groups in each ligand start to 

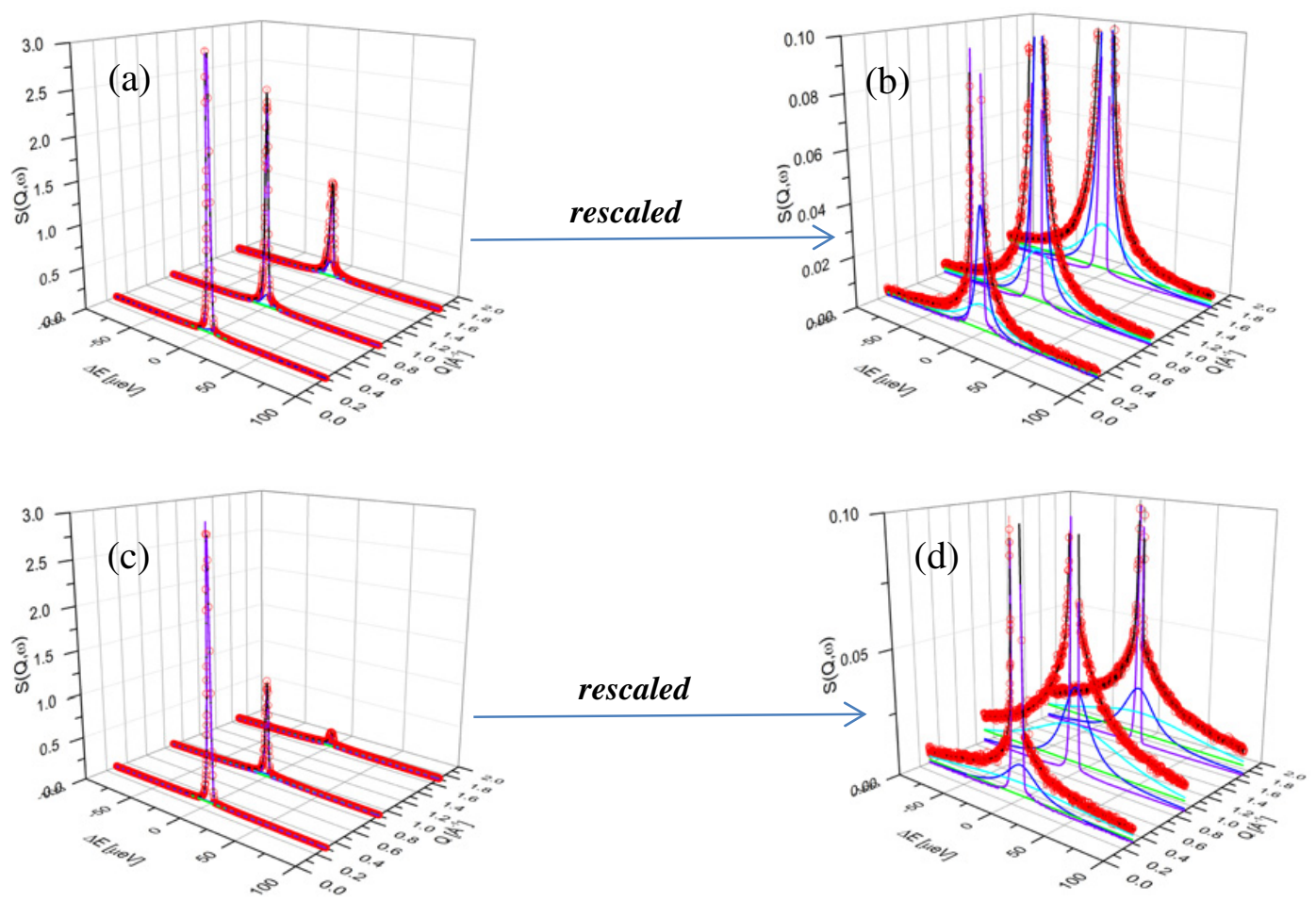

Figure 3. IBU-POSS spectra measured at BASIS (a)\&(b) at $270 \mathrm{~K}$, and (c)\&(d) at $370 \mathrm{~K}$. The red circles represent the experimental data, and the lines are for the fit function (black), elastic peak (purple), quasielastic terms (blue and turquoise) and background (green).

exchange their locations, i.e. they undergo rotation around the axis passing through the O-Si (siloxy) bond. This type of dynamical transition correlates with a structural transition observed in temperature dependent neutron and X-ray powder diffraction measurements [7].

In Fig. 2c the MSD for the DMS-POSS is shown. Similarities to the M-POSS and to the TMS-POSS can be noted consistent with the fact that the two the methyl groups dominate the hydrogen dynamics of the DMSPOSS ligand. Since the DMS-POSS ligand also has a single hydrogen atom attached to the silane along with the two methyl groups, some differences are also encountered. Whereas the rapid increase for the M-POSS and for the TMS-POSS MSDs starts at around $60 \mathrm{~K}$, this increase is seen for the DMS-POSS at slightly lower temperature. Also the MSD values remain a bit lower, which is a direct reflection of the fact that this averaged MSD includes also the contribution from the single hydrogen. However, up to about $150 \mathrm{~K}$ the shape of the MSD curve for DMSPOSS is very similar to that of M-POSS, indicating that the methyl dynamics are similar in these materials, namely rotations around local 3-fold axes. At around 150 and $170 \mathrm{~K}$ discontinuities to higher MSD values can be noted. Both of these steps are signs of dynamical transitions in the sample. As for the TMS-POSS, the transition at around $170 \mathrm{~K}$ can be assigned to rotation around an axis passing through the O-Si (siloxy) bond, in which, the two methyl groups and the hydrogen atom rotate around this axis. Neutron and synchrotron x-ray powder diffraction data and Raman spectra also indicate structural phase transitions at $145 \mathrm{~K}$ and $170 \mathrm{~K}$ in DMS-POSS [7].

The IBU-POSS ligand dynamics appear to be very different from those of the three other samples, as can be noted from the data in Fig. 2d. The MSDs only increase slightly below $180 \mathrm{~K}$, but above this temperature a rapid increase occurs, indicating that observable dynamical processes begin to take place in the sample. This shift of the methyl dynamics to higher temperatures reflects the larger energy barrier to methyl rotation in IBU-POSS, where the methyl groups are attached to carbon atoms, compared to the other three POSS molecules where the methyl groups are instead attached to Si atoms. Another clear dynamical transition in IBU-POSS is observed at about $330 \mathrm{~K}$, which also corresponds to a structural phase transition [2].

Dynamics of these POSS ligands can be observed by QENS measurements. Here we will only discuss the QENS data for the IBU-POSS (Fig. 3). The data for the other samples will be discussed elsewhere [6], [8]. We can clearly note how the elastic intensity decreases as a function of $\mathrm{Q}$, and almost vanishes at higher $\mathrm{Q}$ values above the phase transition temperature. The data at these two temperatures obviously require very different dynamical models for their description. To understand the differences in more detail we have fitted the QENS spectra to the following expression:

$S(Q, \omega)=f\left[p_{0} \delta(\omega)+\sum_{1=i}^{n} p i \frac{1}{\pi} \frac{\Delta_{1}(Q)}{\omega^{2}+\Delta^{2}}\right] \otimes R(Q, \omega)+B$

where $f$ is a scaling factor and $p_{i}$ are the amplitudes of the elastic and quasielastic components. A low temperature data set was used for the instrumental resolution function, $R(Q, \omega)$, which was then convoluted $\otimes$ with the fitted contributions, a $\delta$-function for the elastic contribution and Lorentzian functions with widths $\Delta_{\mathrm{i}}$ for the quasielastic 


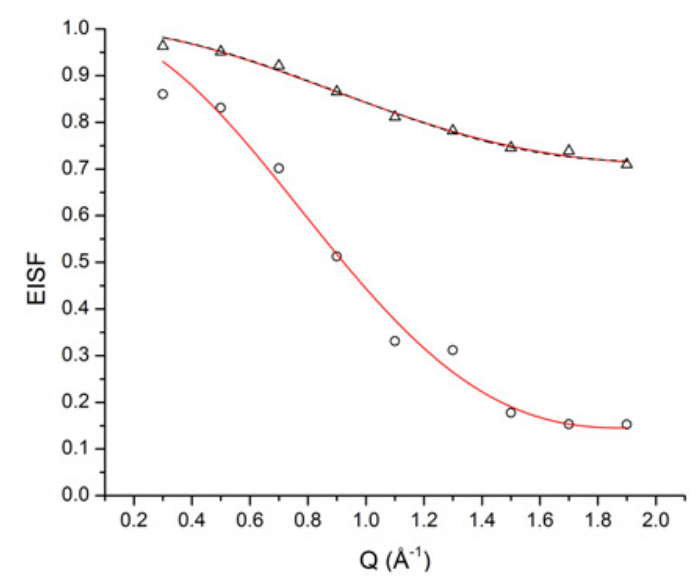

Figure 4. EISF of the broader quasielastic component for IBUPOSS at $270 \mathrm{~K}$ (triangles), and at $370 \mathrm{~K}$ (circles). The red lines represent fits of continuous rotational diffusion, and the dashed black line represents a fit of 3-site jump diffusion model.

contribution. Each spectrum was fit well by using two Lorentzian functions for the quasielastic part. The widths of the Lorentzian components were Q independent, indicating that the observed processes are localized in nature. Their widths, however, were clearly very different from each other, the broad one being about 3 to 4 times wider in energy than the narrow one. Here we will only discuss the broad component. The elastic incoherent structure factor (EISF) for the broad Lorentzian component is shown in Fig. 4 for 270 and $370 \mathrm{~K}$. Two different models were used to describe the experimental points: a model for jump diffusion among 3 equivalent sites on a circle and a model for continuous rotational diffusion on a sphere. The details of both models are described in literature [9] and can be expressed as follows

$$
\begin{gathered}
A_{3 \text { sites }}(Q)=(1-m)+m\left[\frac{1}{3}+\frac{2}{3} j_{0}(Q r \sqrt{3})\right] \\
A_{\text {cont }}(Q)=(1-m)+m\left[(2 l+1) j^{2} l(Q r)\right] ; l=0,1,2, \ldots
\end{gathered}
$$

$m$ is the fraction of mobile hydrogen in the material, $j_{l}$ is the spherical Bessel function of order $l$, and $r$ is the radius of rotation. Only the first term for the continuous rotational jump diffusion was considered $(l=0)$ and the fit parameters were $m$ and $r$. At $270 \mathrm{~K}$ the fraction of mobile hydrogen is $0.35 \pm 0.014$ and $0.29 \pm 0.13$ for the 3 -site jump model and for the continuous diffusion model, respectively. This indicates that about $30 \%$ of the hydrogen atoms participate in the fast motion. The extracted radii of rotation were $1.33 \pm 0.05 \AA$ (3-site jump diffusion) and $1.48 \pm 0.07 \AA$ (continuous rotational diffusion), each somewhat higher than the value expected for methyl rotation limited by the methyl $\mathrm{C}-\mathrm{H}$ bond length (typically around $1 \AA$ ). Considering the fit parameters obtained, neither of these models can describe the methyl dynamics of the IBU-POSS ligands reasonably at $270 \mathrm{~K}$. Therefore we assume that the dynamics also involves other parts of the isobutyl ligand, not only the methyl groups. The data at $370 \mathrm{~K}$ cannot be described by the 3-site jump model, and the continuous rotational diffusion model was used to estimate the fit parameters. It is obvious that at $370 \mathrm{~K}$, a much larger fraction of hydrogen $(0.85 \pm 0.12)$ participates in this motion. In addition, the radius of the rotation increases to $1.68 \pm 0.04 \AA$. However, a more detailed model is necessary to fully understand the geometry of the isobutyl ligand dynamics. The activation energy for the rotational diffusion characterized by the broad Lorentzian component is $11.4 \mathrm{~kJ} / \mathrm{mol}$. This activation energy remains the same below and above the phase transition temperature, but a discontinuity of the quasielastic broadening was observed at the phase transition temperature.

\section{Conclusions}

A number of dynamic transitions associated with the ligands were observed for TMS-POSS, DMS-POSS and IBU-POSS samples, but not for the M-POSS sample, in the temperature range we explored. The complex ligand dynamics are the driving force for structural phase transitions observed in these samples. Methyl group rotations occur at similar temperatures for the ligands of M-POSS, DMS-POSS and TMS-POSS. However, the onset of methyl rotations is shifted to significantly higher temperatures for the isobutyl ligands of IBU-POSS. This is due to the significantly larger rotational energy barriers for methyl groups attached to carbon atoms compared with silicon atoms.

Neutron research at Spallation Neutron Source was sponsored by the Scientific User Facilities Division, Office of Basic Energy Sciences, U. S. Department of Energy. This work utilized facilities supported in part by the National Science Foundation under Agreement No. DMR-0944772. Identification of commercial products does not imply endorsement by the National Institute of Standards and Technology nor does it imply that these are the best for the purpose. The authors would like to thank K.D. Dobbs (DuPont) for the POSS figures.

\section{References}

[1] D.B. Cordes, P.D. Lickiss, and F.Rataboul, Chem. Rev., 110, 2081 (2010)

[2] G. Croce, F. Carniato, M. Milanesio, E. Boccaleri, G. Paul, W. van Beek, L. Marchese. Phys. Chem. Chem. Phys., 11, 10087 (2009)

[3] C. Marcolli, P. Lainé, R. Bühler, G. Calzaferri, J. Tomkinson, J. Phys. Chem. B., 101, 1171 (1997)

[4] E. Mamontov, K.W. Herwig, Rev. Sci. Intsrum. 82, 085109 (2011)

[5] A. Meyer, R.M. Dimeo, P.M. Gehring, D.A. Neumann, Rev. Sci. Instrum., 74, 2759 (2003)

[6] N. Jalarvo, O. Gourdon, G. Ehlers, M. Tyagi, S. K. Kumar, K. D. Dobbs, R. J. Smalley, W. E. Guise, A. Ramirez-Cuesta, C. Wildgruber, M. K. Crawford. J. Phys. Chem. C., 118, 5579 (2014)

[7] M. K. Crawford, P. Whitfield, et al. in preparation

[8] M. K. Crawford, N. Jalarvo, and M. Tyagi et al. in preparation

[9] Bee, M. Quasielastic Neutron Scattering; Adam Hilger: Philadelphia, PA, 1988 